\title{
Des derniers locuteurs aux néo-locuteurs : revitalisation linguistique en Europe
}

\author{
James Costa*
}

\section{INTRODUCTION}

Cette contribution cherche à proposer un ensemble de réflexions sur un type de terrain particulier, celui de ce que l'on pourrait appeler la revitalisation linguistique. Par là, j'entends l'ensemble des pratiques mises en œuvre par divers acteurs sociaux (linguistes, militants par exemple) en vue d'opérer un changement dans la configuration linguistique d'un groupe particulier.

Ce thème est peu présent dans la recherche française, tout comme, de manière plus générale, celui des langues en danger. Mounin (2006 [1992]) faisait remarquer que la problématique était peu développée en France pour trois raisons principales: la confusion qui régnait dans la terminologie; l'étendue d'un domaine qui couvre la documentation de langues en danger comme leur "sauvetage"; l'habitude en linguistique française à ne pas prendre en compte des langues régionales. De fait, si des travaux existent en France, en sociolinguistique sur des problématiques proches de celles abordées par le courant des langues en danger (Lafont, 1984), elles s'intéressent peu aux questions de terrain. C'est vers l'anthropologie qu'il faut alors se tourner (cf. Rulhes, 2000), mais on sait que l'anthropologie française, contrairement à ce qui se passe aux Etats-Unis, s'intéresse peu aux questions de langues.

L'émergence d'un courant de pensée sur les langues en danger, aux États-Unis, en Amérique Latine, en Angleterre, nous permet désormais de repenser certaines problématiques européennes sous un angle nouveau. Car à travers toute l'Europe, des Hautes-Terres d'Écosse aux Balkans, de la Baltique à la Galice, ce sont des 
dizaines de mouvements qui tentent de développer l'usage de langues que l'ont aurait pu considérer comme en voie d'extinction il y a cinquante ans. Si ces mouvements possèdent indubitablement des caractéristiques communes, chacun reste néanmoins unique, spécifiquement lié au contexte social qui lui permet d'exister. Ainsi, certains de ces mouvements sont généralement considérés comme des succès, comme en Catalogne ou au Pays de Galles. D'autres comme des échecs, comme en Irlande. Dans tous les cas, ces questions sont susceptibles de déchaîner des débats parfois violents dans les media, voire dans la rue. Dans tous les cas également, seul un travail de terrain permet de comprendre comment ces mouvements existent localement, comment ils prennent sens pour les divers acteurs qui en sont partie prenante.

Cet article vise donc principalement à faire partager un regard différent sur la question des langues en danger, et de leur prise en compte en sociolinguistique principalement. Ce regard, c'est celui d'un militant, non pas d'une langue à l'origine mais de toutes les langues, devenu enseignant d'occitan en Provence, puis chercheur, nourri des questions liées à un travail de terrain multiforme.

Pour mieux comprendre ce travail de terrain, cet article proposera d'abord une contextualisation permettant de comprendre la spécificité d'un travail de terrain autour des questions de revitalisation en Europe puis en France. Dans un second temps, il s'agira d'illustrer, à travers un parcours singulier, ce travail de terrain, qui a mené à une thèse de sociolinguistique à l'Université de Grenoble sur la revitalisation linguistique. En parallèle, j'ai pu travailler à l'Institut National de Recherche Pédagogique, affecté au laboratoire lyonnais $\mathrm{ICAR}^{1}$, en étroite collaboration avec le laboratoire $\mathrm{DDL}^{2}$.

\section{UNE EUROPE DE LA DIVERSITE?}

Si l'Union Européenne s'est construite sur l'idée qu'il fallait valoriser la diversité linguistique européenne, et si le Conseil de l'Europe a été créé en 1949

\footnotetext{
* Laboratoire ICAR (ENS de Lyon, CNRS et Université de Lyon)

1 Interactions, Corpus, Apprentissages, Représentations, laboratoires lyonnais de linguistique, centré sur l'analyse des interactions

${ }_{2}^{2}$ Dynamique du Langage. Voir les articles de Grinevald, Bert, Gelas, Bon, ce numéro.
} 
pour promouvoir la culture en Europe, jusqu'aux années 1990 il faut entendre l'expression "langues européennes" comme "langues officielles des États membres". De plus en plus néanmoins, sous la pression en particulier de l'Espagne ou du Royaume-Uni, d'autres langues, comme le catalan, le galicien ou le gallois trouvent leur place dans les institutions européennes.

\subsection{Une diversité revendiquée mais toute relative}

L’Union européenne revendique sa diversité et promeut ses langues, symboliquement, par exemple à travers sa devise 'l'unité dans la diversité, et pratiquement, à travers l'adoption par ses membres de textes comme la Charte européenne pour les langues régionales ou minoritaires (Council of Europe, 1992).

Pourtant, cette diversité est toute relative. Avec quelques trois cent langues, l'Europe ne représenterait qu'environ 3\% des langues du monde ${ }^{3}$. Cette diversité est encore plus relative si l'on s'intéresse au nombre de familles de langues présentes en Europe, puisqu'il n'y a que deux familles représentées - les langues indoeuropéennes (la plupart des langues européennes) et finno-ougriennes (hongrois, estonien, finnois, sami) - ainsi qu'un isolat, le basque, survivance de l'Europe pré-indoeuropéenne.

Le fait que l'Europe mette en avant, pour sa communication et dans ses principes, une diversité qui n'a finalement rien d'exceptionnel est donc avant tout un indice d'une volonté de promouvoir une idéologie en apparence au moins plus pluraliste, respectueuse de la diversité linguistique comme principe fondateur de la paix en Europe.

\subsection{Une importante diversité de situations}

Au-delà de cette apparente faiblesse (relative) de la diversité linguistique européenne, on est confronté à une variété de contextes très importante, en termes

\footnotetext{
${ }^{3}$ T. Skutnabb-Kangas (2002) donne le chiffre de 275 langues, en comptant toutes les langues connues d'Europe, y compris les langues qui ont disparu depuis longtemps. Le Summer Institute of Linguistics l'évalue à 300 (Lewis, 2009), et Austin (2008: 276) donne le chiffre de 239 langues approximativement, dont 100 seraient en danger, et 10 presque éteintes. A comparer avec le chiffre donné également par Austin (2008)
} 
de statuts politiques et de vitalité. Il existe des différences considérables entre les contextes d'Europe de l'ouest, marqués par la présence de minorités linguistiques, circonscrites géographiquement, et ceux d'Europe de l'est, marqués par la présence de minorités nationales ou ethniques éclatées en divers groupes dans toute l'Europe centrale et orientale. On trouve ainsi ${ }^{4}$ :

a. Des langues dominantes hégémoniques, officielles en Europe mais aussi dans diverses parties du monde du fait de l'expansion coloniale des États dont elles sont originaires : anglais, espagnol, français, portugais, russe, mais aussi hollandais, italien. D'autres langues nationales sont dominantes sur un espace donné, comme les langues scandinaves, le hongrois, le slovène, etc.

b. Des langues parlées par un nombre important de locuteurs et pourtant menacées, bien connues dans leur forme officielle (certains dialectes peuvent être très mal documentés) : c'est le cas du catalan ${ }^{5}$, mais aussi de l'occitan, parlé selon certaines estimations par trois millions de personnes (Sibille, 2002), mais par des locuteurs désormais âgés, vivant dans un territoire très vaste peuplé par plus de quinze millions de personnes. L'écossais (ou Scots), langue germanique parlée en Écosse, est dans un cas relativement similaire : une enquête de 1996 le donne parlé par 1,5 million de personnes, mais la plupart pensent parler un mauvais anglais. Des emprunts massifs à l'anglais tendent à faire perdre à la langue nombre de ses spécificités. Le romani serait parlé par 1.5 million de personnes selon le Summer Institute of Linguistics (Lewis, 2009).

c. Des langues avec un nombre relativement faible de locuteurs, très menacées comme le breton (200 000, mais un million au début du 20e siècle - cf. Broudic, 2000), les gaéliques d'Irlande (peut-être moins de 30000 locuteurs réels ${ }^{6}$ ) ou d'Écosse (moins de $60000^{7}$ ), ou en situation plus favorable, comme le gallois (600 000), qui a vu le nombre de ses locuteurs augmenter entre les recensements

de 1000 langues pour les Amériques (dont 170 seraient presque éteintes) ou 2092 en Afrique (300 en danger, 46 presque éteintes).

${ }_{5}^{4}$ Pour les langues sans références directes, voir Salminen (2007).

57 à 10 million de locuteurs. Langue officielle en Catalogne, Valence, Baléares, Andorre, mais concurrencée par l'espagnol, et très menacée en Catalogne Nord (la majeure partie des Pyrénées Orientales).

${ }^{6}$ Langue officielle de la République d'Irlande, le gaélique est enseigné obligatoirement à l'école. Près d'1,5 million de personnes déclarent savoir le parler lors des recensements, mais il est difficile de savoir combien la pratiquent effectivement (cf. Price, 2000).

${ }^{7}$ Les chiffres pour l'Ecosse et le Pays de Galles sont ceux du recensement de 2001. 
de 1991 et 2001 grâce à une politique volontariste, mais qui perd des locuteurs dans ses bastions traditionnels ${ }^{8}$, ou encore le basque (600 000), en augmentation également.

d. Des langues ayant presque disparu, comme le vote (une cinquantaine de locuteurs en Estonie), le live (une trentaine de locuteurs en Lettonie) et certains dialectes sámi, en Suède, Norvège ou Finlande.

e. Des langues disparues depuis longtemps : Dalmate (langue romane de la côte croate), norrois (langue scandinave des îles Shetland et Orcades, qui a cessé d'être parlée au $19^{\text {e }}$ siècle (cf. Barnes, 1998)), prussien (langue balte parlée dans le nord de ce qui est aujourd'hui la Pologne), gothique (en Crimée). Certaines ont complètement cessé d'être parlées, mais ont été reconstituées, comme le cambrien dans le nord de l'Angleterre, disparu au $13^{\mathrm{e}}$ siècle $^{9}$, ou apprises par des enthousiastes, comme dans le cas du manx. Le dernier locuteur traditionnel de cette langue, qui avait appris la langue dans son environnement familial, est mort en 1974, mais des dizaines d'heures d'enregistrement existent, et aujourd'hui la langue est enseignée dans plusieurs crèches et écoles (cf. Thomson, 2000). Le cornique avait également disparu : la dernière locutrice est morte en 1777 (mais sur sa tombe on trouve une inscription en cornique, ce qui laisse supposer que la langue a continué à être au moins partiellement connue). La langue a été revitalisée à partir du début du $20^{\mathrm{e}}$ siècle, et aujourd'hui on compte plusieurs centaines de locuteurs (cf. Payton, 2000).

Il faut noter qu'une sous-famille entière, la famille des langues celtiques, est au bord de la disparition (cf. Crystal, 2005). Autrefois parlées dans toute l'Europe, de la Turquie à l'ouest de l'Écosse, les langues celtiques sont désormais cantonnées aux côtes nord-ouest de l'Europe, et à l'heure actuelle, seul le gallois bénéficie d'un regain mesurable et observable.

\subsection{Recherche sur LED en Europe et dans le reste du monde.}

${ }^{8}$ Même si, et c'est un point crucial, le taux de transmission familiale augmente pour la première fois (Office for national statistics :

http://www.statistics.gov.uk/cci/nugget.asp?id=447).

${ }^{9}$ Seuls les chiffres ont survécu jusqu'à nous, à travers des comptines. 
Les langues minoritaires du monde, qui peuvent être ou ne pas être en danger, font l'objet de traditions de recherches différentes selon les terrains et les domaines scientifiques.

On peut dégager quelques grandes différences entre les terrains européens et américains pour essayer de cerner la spécificité du travail en Europe, par rapport à la plupart des autres travaux ici qui permettent de cerner les contextes américains. Si postuler que l'on ne peut travailler de la même manière en Amérique et en Europe semble une évidence, il peut être intéressant de montrer en quoi consistent quelques unes de ces différences, en termes d'approches de terrain comme en termes de contextes, d'histoires collectives.

Pour F. Grin (2003: 174), économiste suisse, spécialisé dans l'économie des langues, on peut classer les contextes de langues minoritaires dans le monde en quatre catégories, qu'il schématise sous forme de trèfle à quatre feuilles ("the diversity clover"), reproduit ci-dessous en français sous forme de tableau :

\begin{tabular}{|c|c|c|c|}
\hline & Contexte & $\begin{array}{c}\text { Localisation } \\
\text { principale }\end{array}$ & $\begin{array}{c}\text { Domaines } \\
\text { scientifiques } \\
\text { produisant un } \\
\text { discours sur ces } \\
\text { domaines }\end{array}$ \\
\hline $\begin{array}{c}\text { Feuille } \\
1\end{array}$ & $\begin{array}{l}\text { Minorités } \\
\text { nationales, } \\
\text { droit des } \\
\text { minorités }\end{array}$ & Europe Orientale & $\begin{array}{c}\text { Relations } \\
\text { internationales, droit } \\
\text { international, sciences } \\
\text { politiques }\end{array}$ \\
\hline $\begin{array}{c}\text { Feuille } \\
2\end{array}$ & $\begin{array}{c}\text { Minorités } \\
\text { linguistique, } \\
\text { revitalisation }\end{array}$ & $\begin{array}{c}\text { Europe } \\
\text { Occidentale }\end{array}$ & $\begin{array}{c}\text { Sociolinguistique, } \\
\text { linguistique appliquée, } \\
\text { sciences de l'éducation }\end{array}$ \\
\hline $\begin{array}{c}\text { Feuille } \\
3\end{array}$ & $\begin{array}{l}\text { Minorités } \\
\text { immigrées }\end{array}$ & $\begin{array}{l}\text { Centres urbains } \\
\text { en Europe et } \\
\text { Amérique du } \\
\text { Nord }\end{array}$ & $\begin{array}{l}\text { Sociologie, sciences de } \\
\text { l'éducation, économie }\end{array}$ \\
\hline Feuille & Peuples & Amériques, & Anthropologie, \\
\hline
\end{tabular}




\begin{tabular}{|c|c|c|c|}
\hline $\mathbf{4}$ & indigènes & $\begin{array}{c}\text { Afrique, } \\
\text { Australasie }\end{array}$ & $\begin{array}{c}\text { ethnologie, études sur } \\
\text { le développement }\end{array}$ \\
\hline
\end{tabular}

Certains aspects de ce tableau peuvent être discutés : en ce qui concerne les domaines scientifiques assignés à chaque contexte par exemple, la sociolinguistique est désormais très présente sur le terrain des peuples indigènes, et la linguistique n'est pas mentionnée. D'autre part, les situations d'Afrique et d'Amériques pourraient être traitées séparément (Grinevald, 2006). Il reste néanmoins tout à fait pertinent que le découpage du champ des langues minoritaires, ou de la diversité linguistique, en plusieurs catégories bien distinctes souligne bien l'absence de contacts réguliers entre spécialistes. Comme l'écrit Grin :

La plupart du temps, les chercheurs appartenant à l'une ou l'autre feuille n'ont que peu d'occasions de rencontrer des chercheurs travaillant sur une autre feuille. C'est encore plus vrai des praticiens sur le terrain (non universitaires) (2003: 175). ${ }^{10}$

Le classement donne aux langues indigènes (sans distinction de continent) une place à part. Or ce sont ces langues, en particulier celles d'Amériques et d'Australie, qui ont servi de base à la réflexion au champ des langues en danger (cf. la collection d'articles de Hale, Krauss, Watahomigie, Yamamoto, Craig, Jeanne \& England, 1992). Les langues européennes sont ainsi souvent exclues de ce champ, et traitées du point de vue de la politique linguistique.

\subsection{Contrastes et similarités : Europe et Amérique Latine}

Un certain nombre de facteurs contribue à distinguer les situations des langues en danger en Europe et en Amérique Latine. Ainsi :

a. Les langues européennes et leurs développements historiques sont relativement bien connus. Il existe des traditions linguistiques et

\footnotetext{
${ }^{10}$ Ma traduction.
} 
sociolinguistiques pour l'ensemble des langues romanes, pour les langues celtiques, et les premiers travaux sur l'attrition linguistique proviennent d'études sur des langues européennes (Dorian, 1981; Sasse, 1992). Elles font par ailleurs souvent l'objet de l'attention de linguistes ou d'historiens amateurs, qui publient à leur sujet.

b. Elles sont écrites (et bien souvent décrites) depuis longtemps pour beaucoup d'entre elles, ce qui n'est que rarement le cas en Amérique. Une littérature écrite est souvent disponible relativement aisément pour divers publics (enfants, adolescents, adultes etc.). Il est de fait plus facile de les introduire dans les divers systèmes éducatifs européens, qui requièrent généralement des formes linguistiques codifiées, standardisées, suivant en cela le modèle des langues dominantes.

c. En Europe, on trouve un nombre important de langues dites collatérales (cf. Eloy, 2004b; Eloy \& Ó hIfearnáin, 2007), c'est-à-dire "des variétés proches objectivement et subjectivement - aux plans linguistique, sociolinguistique et historique, les variétés tendanciellement en contraste étant historiquement liées par les modalités de leur développement" (Eloy, 2004a: 10) (occitan et français, ou catalan et espagnol par exemple). La situation européenne, avec une famille de langues largement majoritaire, la famille indo-européenne, rappelle par ailleurs ce que l'on peut observer en Afrique (avec par exemple le large complexe bantou ${ }^{11}$ ), alors qu'en Amérique, on trouve un nombre considérable de familles de langues, cohabitant parfois dans un espace très restreint ${ }^{12}$.

d. Les contextes historiques sont différents : en Europe, on peut considérer que toutes les langues présentes, majoritaires ou minoritaires, sont indigènes (hors langues de l'immigration récente), ou du moins que la distinction entre langues indigènes et langues importées n'a pas de sens pour décrire les situations minoritaires de langues en danger. En Amérique, il existe une opposition nette entre langues indigènes et langues de colonisation. La notion de langue indigène ou même autochtone est cependant loin d'être clairement définie : ainsi Spolsky

${ }^{11}$ Voir le chapitre de S. Manus sur le makondé du Mozambique et de Tanzanie et celui de H. Gelas sur le gisir du Gabon, chapitres traitant de langues de la même famille bantoue. 
(2008: 153) fait-il remarquer que l'espagnols étaient parlé dans le sud-ouest des États-Unis avant le navajo.

e. Ces enjeux historiques conditionnent les positionnements du chercheur sur le terrain, en le plaçant au cœur d'une dynamique de colonisation/décolonisation en Amérique Latine, voire de conditions historiques particulièrement douloureuses, comme les génocides dont ont été victimes les populations indigènes sur tout le continent.

f. Les enjeux diffèrent d'un continent à l'autre: si en Europe il s'agit principalement, au moins en apparence, de revendications linguistiques et du droit à parler une langue, voire d'enjeux patrimoniaux dans certains cas, en Amérique Latine en particulier la langue est souvent liée à d'autres revendications : citoyenneté, égalité sociale, questions territoriales.

g. L'Europe, du fait de l'existence d'États-nations depuis plus de deux siècles, présente un visage relativement uniforme, au moins en surface, en termes d'idéologies linguistiques, contrairement aux Amériques, où l'on observe une grande variété de situations (cf. Kroskrity \& Field, 2009).

h. En Europe, l'alphabétisation et les droits concédés aux minorités au sortir de deux guerres mondiales peuvent donner au moins l'apparence d'un plus grand respect de la diversité linguistique et culturelle. L'existence de cette diversité est acceptée au moins en principe par les instances internationales européennes (Union européenne et Conseil de l'Europe). De plus, certaines langues minoritaires sont enseignées depuis longtemps. Celles-ci sont présentes dans les media et sur internet : on trouve de nombreux sites en langues minoritaires européennes alors que pour le moment on trouve surtout des sites sur les langues indigènes d'Amérique.

Ces quelques indications demeurent toutefois superficielles, par manque de place, mais aussi par manque d'information sur l'ensemble des situations linguistiques et micro-sociolinguistiques en Europe comme en Amérique Latine. Pourtant, dans le travail sur le terrain, avec les locuteurs, leurs attentes, leurs

\footnotetext{
${ }^{12}$ Voir par exemple les chapitres de S. de Lancey et de M. Mithun pour les nombreuses familles de langues
} 
espoirs, leurs représentations, mais aussi dans les écoles avec les enfants, de nombreux parallèles permettent de rapprocher les situations européennes des situations amérindiennes. Les contextes différents certes en surface, mais la réalité sous-jacente, les expériences individuelles, la détresse que l'on peut rencontrer chez les derniers locuteurs, le plaisir que l'on peut observer chez les nouveaux locuteurs, sont autant de points communs à souligner. Ainsi, le fragment de discours que l'on trouve dans l'extrait suivant, qui est celui d'une enseignante d'une langue de Californie, n'est-il pas sans rappeler certains discours d'enseignants en Europe :

When I go into the classroom, sometimes I look at the kids that I'm teaching, and I see the potential, and I see the desire that they have, and it's like I'm holding this very fragile, precious thing in my hand ${ }^{13}$ (citée dans Hinton, 1996: 190).

A ce titre, les langues minoritaires européennes sont aussi des "langues en danger" au sens où l'entendent les contributeurs de ce volume; elles risquent tout autant de disparaître du paysage linguistique mondial, et elles prennent part, de plus en plus, à la formation d'un discours global sur cette problématique, notamment autour de la question de la préservation de la diversité linguistique comme bien commun de l'humanité. En ce sens la fragilité de la diversité européenne est à rapprocher de la fragilité sud ou nord américaine. Si les langues n'existent pas en dehors des pratiques des locuteurs, ces pratiques sont en régression, pour une multiplicité de raisons qui ne tiennent pas toutes aux impératifs résultant de la nécessité d'une meilleure communication, ou à la fatalité de la globalisation culturelle.

Le cas de la France, et de ses langues, est à ce titre particulièrement emblématique de cette récession des pratiques linguistiques, et conjugue une multiplicité de facteurs, idéologiques, historiques, géographiques (Encrevé, 2002).

du nord-ouest des Etats-Unis.

${ }^{13}$ Quand j'entre dans la classe, parfois je regarde les enfants à qui j'enseigne, et je vois le potentiel, et je vois leur désir, et c'est comme si je tenais dans ma main cette chose très fragile, très précieuse [le futur de la langue]. 


\section{LA FRANCE ET SES LANGUES}

\subsection{Le complexe français}

Devant le déclin international du français, la France a eu tendance à miser, dans sa politique de Francophonie, sur une stratégie de promotion du plurilinguisme et de la diversité culturelle - en restreignant toutefois ce champ aux langues et cultures officielles des États (cf. Costa \& Lambert, 2009). On sait la difficulté que la France a à accepter la diversité linguistique présente sur son terrain, et ce depuis près de 500 ans. Le monolinguisme semble constitutif de la construction d'une identité française spécifique. En fait, la situation actuelle résulte d'une série de choix particuliers, liés à chaque fois au contexte politique de l'époque à laquelle ils sont effectués.

La France est pourtant l'un des pays européens les plus divers linguistiquement, avec sur son territoire métropolitain plus d'une dizaine de langues dites "régionales", en plus du français, et trois sous-familles de langues (romane, celtique et germanique) et un isolat, le basque, qui ne semble pouvoir être rattaché à aucune autre langue.

En termes de désignation, et concernant la France métropolitaine, le discours politique a longtemps parlé de "langues locales" et surtout de "patois" pour désigner les langues des anciennes provinces de l'ancien régime (cf. Gardy, 1990). On rencontre aussi l'expression de "langues provinciales". Une des forces de la politique linguistique française a toujours été d'éviter de nommer ces langues individuellement. Aussi l'article 2 de la Constitution française, qui dispose que la langue de la République est le français, reprend-il le même procédé que l'Edit de Villers-Cotterêts (1539) en imposant le français sans explicitement interdire nommément aucune autre langue. Celles-ci sont ainsi implicitement renvoyées dans une zone de non existence.

\subsection{Des langues régionales...}

Ces langues cohabitent avec le français dans le cadre d'une importante variété de contextes (cf. Sibille, 2002) : ainsi, le breton, langue celtique, n'est-il parlé 
qu'à l'intérieur d'un territoire relativement peu étendu, à l'extrémité ouest de la péninsule armoricaine, sur un département entier et deux demi-départements : le basque est parlé dans un demi-département alors que l'occitan (ou langue d'oc) est parlé, sous une importante variété de formes, dans trente-trois départements. Si le basque (44 000 locuteurs en France ${ }^{14}$ ) et le catalan (132 000 en France) n'ont aucun statut en France, ces langues sont reconnues comme langues officielles régionales en Espagne, et les locuteurs du côté français bénéficient de certaines infrastructures présentes au sud de la frontière, radios et télévisions en particulier, ainsi que du dynamisme régional espagnol. L'occitan (entre 526000 et 3 millions ${ }^{15}$ ) est surtout parlé en France, mais il est reconnu comme langue officielle dans une vallée en Espagne et dans onze vallées italiennes. Le flamand (10 000), parlé dans l'arrondissement de Dunkerque, est reconnu en Belgique et sous sa forme hollandaise est la langue officielle des Pays-Bas. Le corse (122 000), qui a longtemps été considéré comme un dialecte de l'italien, est aujourd'hui vu comme une langue autonome, et est enseigné comme tel en Corse où il jouit d'un statut particulier au sein de la Collectivité Territoriale de Corse. L'alsacien (548 000) est quant à lui souvent considéré comme un dialecte allemand $^{16}$. Le francoprovençal (70 000), parlé en France, au val d'Aoste en Italie et en Suisse, n'a été identifié comme langue autonome qu'en 1873 par un linguiste italien, G. I. Ascoli (cf. Tuaillon, 1972). Enfin, le breton (304 000) est le dernier représentant continental des langues celtiques, et le cornique, parlé en Cornouailles britanniques (Sud-ouest de l'Angleterre), seule langue avec laquelle une intercompréhension était peut-être possible, s'est éteint au $18^{\mathrm{e}}$ siècle. Il est certes relativement proche linguistiquement du gallois, dont le nombre de

\footnotetext{
${ }^{14}$ Les chiffres donnés pour l'ensemble de ces langues sont ceux de l'enquête INSEE-INED de 1999, rapportés par Héran, Filhon \& Deprez (2002). Ils sont à prendre avec précaution, tant pour des questions de méthodologie que pour des questions d'interprétation (cf. Martel, 2007a).

${ }^{15}$ Le chiffre de trois millions de locuteurs est celui donné par Sibille (2002). L'imprécision des données illustre bien le problème qu'il y a à comptabiliser des locuteurs d'une langue dont ses propres locuteurs ont souvent honte, ou qu'ils estiment mal parler par rapport à une norme immanente qui serait représentée par la 'bonne littérature', ou par un parler prestigieux : ainsi en Provence la région d'Arles est réputée parler un "bon provençal", alors que les locuteurs des régions plus septentrionales vont estimer ne parler qu'un patois. À l'inverse, certains auront tendance à surestimer leurs compétences pour des raisons sentimentales ou de valorisation d'une identité qu'ils perçoivent comme leur appartenant de droit. Certains locuteurs manquent par ailleurs de personnes avec qui converser dans cette langue et ne peuvent pas ou plus évaluer leur compétence réelle.

16 Ainsi, l'Éducation nationale considère que la forme standard du dialecte, celle qui doit être enseignée à l'école, est l'allemand standard.
} 
locuteurs croît désormais, mais géographiquement éloigné. Les langues d'oïl (gallo, picard, lorrain, berrichon etc.) ont connu elles des fortunes diverses, vivant souvent dans l'ombre de la plus prestigieuse d'entre elles, le français (mais Manzano, 2005: considère que c'est cette proximité qui leur assure une vitalité plus importante que les études ne le montrent habituellement). La définition même de ces langues pose souvent problème, de même que leur nomination et leur écriture (l'occitan dispose par exemple, en Provence, de deux systèmes graphiques concurrents).

\section{3. ...aux langues de France}

En 1999, le gouvernement cherche à faire adopter la Charte européenne des langues régionales ou minoritaires, un texte de 1992 émanant du Conseil de

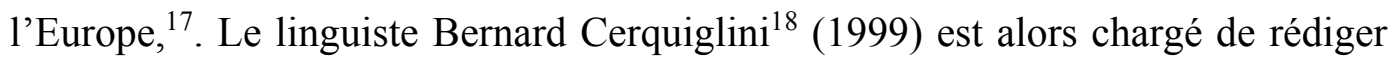
un rapport sur les langues parlées sur l'ensemble du territoire national: il en identifie près de quatre-cents. A partir de cette liste, soixante-quinze d'entre elles seront répertoriées comme "langues de France", catégorie nouvelle inventée à cette occasion ${ }^{19}$. Ces langues sont regroupées en cinq catégories :

- les langues régionales territorialisées (celles dont il a été question ci-dessus);

- des langues non-territorialisées présentes depuis longtemps sur le territoire, comme le romani ou le yiddish;

- les langues des départements, territoires et pays d'Outre-mer;

- la langue des signes française;

- certaines langues de l'immigration non reconnues sur leur territoire d'origine, comme l'arabe dialectal, le berbère ou l'arménien occidental.

Ce chiffre de soixante-quinze langues a été par la suite utilisé dans les media par les détracteurs de la reconnaissance officielle de langues autres que le français en France comme un argument allant dans leur sens : devant l'impossibilité de

\footnotetext{
${ }^{17}$ Le gouvernement français a signé ce texte en 1999, mais le parlement ne l'a à ce jour pas ratifié. ${ }^{18}$ Voir http://www.culture.gouv.fr/culture/dglf/lang-reg/rapport_cerquiglini/langues-france.html
} 
reconnaître autant de langues au même niveau, le statu quo serait la meilleure solution, et la meilleure manière de garantir l'unité de la nation. Un tel argument fait cependant abstraction de la diversité des contextes, et de la variété des revendications : il n'est par exemple pas question, dans la revendication militante, de rendre obligatoire aucune de ces langues dans l'administration ou la justice. Par contre, une connaissance du créole pourrait s'avérer un atout considérable pour les fonctionnaires travaillant dans certains territoires d'Outre-mer.

\subsection{Du mépris à une ébauche de reconnaissance}

Historiquement, ces langues ont également connu des évolutions très différentes, souvent liées au sort politique des pays ou provinces où elles étaient parlées: ainsi, le basque, véritable miraculé de la diffusion des langues indoeuropéennes, est écrit depuis le $16^{\mathrm{e}}$ siècle, alors que l'occitan s'écrit depuis plus de 1000 ans et a servi de véhicule à la prestigieuse littérature des Troubadours au Moyen-Âge. L'occitan a par ailleurs longtemps été langue administrative écrite, alors que la cour du roi de France employait le latin. La matière de Bretagne, cycle littéraire auquel appartiennent par exemple les romans du Graal, écrite en partie en gallois et en breton, a inspiré une grande partie de la littérature française médiévale (Cerquiglini, 2003).

Si la diversité des langues parmi le peuple ne pose pas réellement de problèmes à la monarchie française, le roi fait néanmoins adopter un édit en 1539, à VillersCotterêts, visant à faire du français la seule langue des administrations du royaume ${ }^{20}$. L'édit vise avant tout le latin, mais aussi l'occitan. Les chancelleries régionales se conforment à l'édit plus ou moins rapidement selon les provinces, avec plus ou moins de bonne volonté (Lodge, 1993).

La Révolution fait d'abord traduire ses textes officiels dans diverses langues du royaume, mais la Terreur, comme les dictatures en général, voit d'un mauvais œil

\footnotetext{
${ }^{19}$ Adoptée depuis par le ministère de la culture et la Direction Générale à la Langue Française et aux Langues de France (DGLFLF).

${ }^{20}$ Soit quelques années seulement après l'adoption d'un texte, adopté en Angleterre, et interdisant l'usage du gallois dans l'administration. A la différence de ce texte, l'Edit de Villers-Cotterêts ne nomme aucune langue autre que le français, ce qui sera un des éléments clefs de la politique linguistique française : les langues concurrentes du français ne sont jamais nommées, on ne les fait ainsi jamais exister. On se contente d'imposer le français.
} 
ces langues qui ne sont pas celles de la capitale. La République n'étant qu'une, la langue devra désormais n'être qu'une, et l'abbé Grégoire lance une enquête sur les 'patois', dont il tirera en 1704 un rapport concluant à la nécessité de les détruire. Les divers régimes qui viendront auront vis-à-vis de ces langues une politique étonnamment homogène d'éradication (Balibar, 1985).

La pratique des langues régionales de France est en déclin depuis le $19^{\mathrm{e}}$ siècle. Un déclin qui s'est accéléré avec l'introduction de la scolarité obligatoire en français à partir de 1882, et particulièrement après la première guerre mondiale, qui a eu des répercussions considérables en termes de démographie et de circulation des personnes. Pourtant, dans le même temps, dès le milieu du $19^{\mathrm{e}}$ siècle, se développent autour des langues de la métropole un certain nombre d'initiatives visant à les protéger, les sauvegarder ou les promouvoir. Le Félibrige, association de poètes provençaux formée autour de Frédéric Mistral, se développe ainsi à partir de 1854 dans l'ensemble du Midi, avec l'ambition de restaurer la langue d'oc (cf. Calamel \& Javel, 2002), et une pétition est mise en place en 1870 en vue de demander un enseignement du breton (cf. Martel, 2005). C'est à ce moment que se fixent des grands récits autour des langues de France, véritables mythes destinés à les légitimer face à l'appareil mythologique du français. Ceuxci sont toujours en vigueur au sein des mouvements de défense de ses langues.

Quelques unes des langues de France feront finalement une timide entrée dans la loi en 1951, avec la loi Deixonne, qui permet leur enseignement, à titre optionnel, en dehors des heures de cours obligatoires (Martel, 2007b). Seules quelques langues figurent nommément - et pour la première fois - dans $1 \mathrm{la} 1 \mathrm{li}^{21}$, le francoprovençal en est par exemple exclu. Le corse et l'alsacien seront ajoutés plus tard, en 1975, et les créoles des DOM-TOM plus récemment encore. Face au recul des pratiques, l'enseignement constitue de fait un domaine clef des stratégies de récupération linguistique mises en place par les mouvements militants en faveur de ces langues.

$\mathrm{Si}$, depuis, ces langues ont connu plusieurs avancées concernant notamment leur enseignement, cette crispation autour de la diversité est toujours très vive, comme

${ }^{21}$ Basque, breton, catalan, langue d'oc. 
en témoignent les débats autour de l'adoption d'une motion reconnaissant simplement leur existence dans la Constitution au printemps 2008. Cette reconnaissance, ne concernant que les seules 'langues régionales', sans plus de précision, et n'impliquant aucun engagement supplémentaire de la part de l'état, sera finalement adoptée, non plus à l'article 1, comme initialement adopté par l'Assemblée Nationale, mais à l'article 75, celui portant sur les collectivités territoriales $^{22}$. C'est néanmoins, symboliquement, un événement considérable. Aujourd'hui, malgré l'état de déclin avancé des pratiques dans ces langues, plusieurs dizaines de milliers d'élèves sont concernés par un enseignement de / en langue régionale, en particulier en corse, en breton, en alsacien et en occitan. Les utiliseront-ils un jour? Cette décision leur appartient. Pourtant, plusieurs régions françaises (Aquitaine, Bretagne, Corse, mais aussi Rhône-Alpes ${ }^{23}$ ) ont par ailleurs récemment initié des mesures de politique linguistique en faveur des langues parlées sur leur territoire, en vue de les revitaliser.

\section{L'ENSEIGNEMENT DES LANGUES REGIONALES - REGARD PORTE A PARTIR DU} TERRAIN

Traditionnellement, les mouvements militants agissant en faveur de la revitalisation des langues européennes ont eu tendance à privilégier une approche se concentrant sur l'enseignement. L'école, lieu symbolique de l'imposition de la langue dominante dans ce discours, se voit ainsi investie du rôle de régénération du groupe par l'enseignement de la langue traditionnelle aux enfants.

Cette attitude est graduellement en train de changer, en faveur d'une plus large prise en compte des questions de transmission familiale (Costa, 2010), mais l'école continue d'être un lieu symbolique à investir (Burban \& Lagarde, 2007), soulignant ainsi la prégnance d'idéologies linguistiques centrées sur une vision des langues basée sur l'écrit.

Dans le contexte esquissé jusqu'ici, je souhaite montrer quelques aspects de travaux en terrain scolaire en France, qui permettront de comprendre à la fois

\footnotetext{
${ }^{22}$ L'article 75.1 dispose ainsi que "Les langues régionales appartiennent au patrimoine de la France". ${ }^{23}$ Voir l'article de Michel Bert, ce volume.
} 
comment les idéologies monolingues dominantes en Europe se manifestent, et comment certains acteurs de terrain leurs résistent (Jaffe, 2008). Pour cela, je brosserai quelques portraits d'acteurs de terrain, enseignants, parents, élèves, en me concentrant sur mon expérience en terrain provençal, d'abord comme enseignant et militant, puis comme chercheur. Ces trois postures me permettent d'avoir trois regards différents sur un même terrain, tout en conservant, au cours des analyses, le regard critique qui sied à l'ethnographe, qui permet un retour réflexif sur les périodes de militantisme et d'enseignement.

\subsection{L'enseignant : une liberté pédagogique encadrée par des contraintes}

Jadis destructeur de langue, ex-hussard noir de la République, l'enseignant est aujourd'hui celui par qui la réparation historique doit venir. Chargé (par l'État, qui le paye, mais aussi, dans le discours militant, par la communauté tout entière) de lutter contre l'effacement de la langue, contre l'idéologie dominante, son personnage est aussi vu comme une véritable figure de lutte et de résistance. A la fois garant de la norme, des parlers authentiques, connaissant l'ensemble des dialectes d'oc et de leurs graphies, connaissant la langue médiévale ${ }^{24}$, il est souvent critiqué pour sa langue, jugée artificielle dans les milieux puristes. Devant être capable de s'intégrer dans la vie d'un établissement scolaire et de faire accepter sa matière comme normale auprès des autres enseignants, il est généralement chargé d'enseignement dans deux, trois ou quatre établissements.

Généralement en charge du recrutement de ses élèves dans ces divers lieux, il doit être capable de convaincre parents et enfants du bien fondé de leur enseignement, puis de leur démarche, devant être capable de garder les élèves d'une année sur l'autre, on attend de lui qu'il s'engage dans le militantisme pour l'enseignement et pour la langue. Lorsque l'école devient le seul lieu de transmission de la langue, l'enseignant est, en d'autres termes, objet d'un surinvestissement symbolique et matériel.

Lui-même ne vient jamais à ce métier sans un bagage personnel, une raison qui le pousse à s'intéresser à cette question de la langue minoritaire. Il ne vient jamais 
non plus sans des attentes, des ambitions qui lui sont propres, un objectif. Souvent également, l'enseignement d'une telle langue s'intègre dans ce qui ressemble à une quête : quête des origines, de soi-même.

Dans mon cas, ayant grandi entre deux langues, le français et l'anglais, entre France et Grande-Bretagne, mais aussi, entre Tahiti, Écosse, Bretagne et Provence, pour suivre des parents enseignants, c'est un attachement profond et précoce aux langues qui m'a poussé à suivre des études de langues celtiques (Celtic studies: gallois, mais aussi irlandais et breton) à l'université d'Aberystwyth, au Pays de Galles. Au gré des rencontres, humaines et livresques, je me suis par la suite investi dans l'apprentissage de l'occitan, dans sa variété provençale, du catalan, puis de l'espagnol.

Ayant enseigné le gallois pendant les deux premières années du troisième millénaire à l'Université de Haute-Bretagne, à Rennes, j'ai ensuite décidé de passer un CAPES d'occitan, afin de promouvoir cette langue en Provence, où j'avais vécu quelques années. Si l'enseignement du gallois constituait un enseignement de langue étrangère, je souhaitais enseigner une langue "locale", pour rendre à des enfants une langue que je considérais leur appartenir de droit. Conception naïve mais romantique, inspirée d'un poème du catalan Salvador Espriu $^{25}$, mais tout à la fois tentative de comprendre ce que signifiait perdre et gagner une langue, une culture, dans une conception largement essentialiste de la langue, inspirée largement, in fine, des conceptions de la langue dans l'idéologie des État-nations.

Travailler sur le terrain, que ce soit avec les derniers locuteurs d'une langue ou avec les futurs nouveaux locuteurs de cette même langue n'est jamais chose facile. La principale difficulté consiste à concilier ses objectifs propres avec la réalité du terrain, qu'elle soit institutionnelle ou personnelle.

Les contraintes du terrain sont nombreuses, et elles s'imposent toujours au praticien, enseignant, chercheur ou autre. Il n'y a rien à gagner à la confrontation avec les autorités locales ou avec les locuteurs eux-mêmes. Malgré tout, la tension

\footnotetext{
${ }^{24}$ Le CAPES d'occitan-langue d'oc prévoit une épreuve de traduction de l'occitan médiéval vers le français.
} 
est systématiquement au cœur d'une telle recherche, les différents acteurs ne pouvant rechercher la même chose. Si l'enseignant cherche à transmettre une langue, parents, institution et élèves ont bien souvent des motivations et des attentes différentes, qui contraignent la pratique enseignante.

\subsection{L'institution : une contrainte insurmontable}

Officiellement, l'institution cherche à "former des locuteurs", la "reconquête d'usages linguistiques, le renouvellement des représentations et des références" ${ }^{26}$. L'objectif affiché est donc ambitieux, compte tenu des moyens généralement disponibles. Il s'agit non seulement d'enseigner une langue, mais de viser une pratique effective dans un cadre idéologique qui dépasserait celui du "patois" associé à la seule ruralité et au passé.

L'institution scolaire doit naviguer entre principes européens et idéologie nationale, entre impératifs budgétaires et nécessité de montrer une ouverture aux minorités. Elle est à la fois lointaine et proche. Lointaine lorsqu'elle impose des programmes, des horaires, des principes, qui semblent à la fois contraignants et avantageux, et proche lorsqu'il s'agit de négocier avec un chef d'établissement l'application de textes rédigés sans connaissance du terrain et sans fournir les moyens financiers pour les faire respecter. Si en France, les instructions du Bulletin officiel de l'Education nationale (B.O.E.N) prévoient un minimum de deux heures de langue régionale hebdomadaire pour les enfants qui choisissent la langue régionale de manière optionnelle au collège (ce qu'ils peuvent faire dès la $6^{\mathrm{e}}$ ), dans mon cas, cet horaire n'a été respecté que pour une seule classe, dans un établissement de la Drôme.

Dans les autres cas, je ne voyais les élèves qu'une seule fois par semaine, c'està-dire que j'enseignais en fait quatorze classes différentes dans la semaine, dans quatre établissements situés dans quatre villages séparés par une distance maximale de 60 kilomètres: Pierrelatte, Saint-Paul-Trois-Châteaux, Suze-la-

${ }^{25}$ [...] Però hem viscut per salvar-vos els mots, / per retornar-vos el nom de cada cosa [...]. [...] (Mais nous avons vécu pour vous sauver les mots, / Pour vous rendre le nom de chaque chose [...]. Salvador Espriu, Inici de Càntic en el Temple.

${ }^{26}$ Langues régionales pour le collège, "Annexe 5, Occitan-Langue d'Oc), B.O.E.N. n 10, 4. Octobre 2007, 88-101. 
Rousse et Nyons. Si une telle distribution faisait sens d'un point de vue comptable, la notion de revitalisation prenait dans ce contexte un sens légèrement différent de ce que j'avais anticipé. Il s'agissait surtout de sensibiliser les élèves à l'existence d'une autre langue, qui n'était plus parlée autour d'eux que par la génération de leurs grands-parents (au mieux), en espérant qu'ils auraient plus tard l'occasion d'approfondir leurs connaissances par eux-mêmes, à l'aide peutêtre des quelques clefs que je pouvais leur donner.

Les contraintes ne sont néanmoins pas seulement institutionnelles (et budgétaires). Elles viennent également des attentes et des motivations des personnes qui gravitent autour de l'entreprise de revitalisation, en particulier les parents d'élèves, et les élèves eux-mêmes.

\subsection{Attentes des parents}

Il faut distinguer les motivations des parents pour les classes bilingues et pour les cours optionnels. Pour les classes bilingues, compte tenu de l'âge auquel les élèves sont inscrits, ce sont les parents qui décident seuls. Il s'agit alors principalement de faire en sorte que son enfant soit bilingue, et souvent on se rend compte lors d'entretiens avec les parents que le choix de la langue elle-même importe peu. Qu'il s'agisse de breton ou de hongrois, ou a fortiori d'anglais, le choix aurait été le même.

Une autre motivation réside dans l'opportunité de pouvoir choisir un établissement scolaire. Souvent, envoyer son enfant en classe bilingue permet d'obtenir une meilleure école, ou, dans le cas d'écoles associatives par immersion, une école plus proche. Le choix de la pédagogie (pédagogie Freinet par exemple dans le cas des Calandretas occitanes) est également important.

Pour les cours de type classique, calqués sur le modèle de l'enseignement des langues étrangères (soit entre une et quelques heures par semaine) les arguments du choix sont autres, d'autant que, les cours s'adressant à des enfants plus âgés $\left(10 / 11\right.$ ans en $\left.6^{\mathrm{e}}\right)$, ceux-ci sont souvent consultés lors de la décision de suivre ou non cet enseignement. Dans mon expérience, les arguments qui persuadent les parents d'inscrire leur enfant en cours de langue régionale sont de deux sortes : 
— la langue régionale peut être un moyen d'ouverture vers des langues proches (comme l'espagnol pour l'occitan, l'allemand standard pour l'alsacien), ou même l'anglais pour le breton.

— une connaissance de la langue régionale peut servir à améliorer le niveau des élèves en français.

Ces arguments n'ont rien de nouveau, ils étaient déjà présents dans la pétition en faveur des langues provinciales présentée au gouvernement français en 1870 (cf. Martel, 2007b), qui fut sans effet du fait de la défaite face à la Prusse.

Certains parents ont toutefois des motivations liées à la langue elle-même. Quelques uns la voient comme une possibilité de s'intégrer dans une région où ils arrivent, tandis que pour d'autres, la langue régionale permettra une communication privilégiée avec les grands-parents. En 2006, lors d'une réunion de présentation de l'option aux parents des futurs élèves de $6^{\mathrm{e}}$, une mère d'élève est venue me voir. Elle était ravie qu'on puisse apprendre l'occitan au collège : son fils allait enfin pouvoir parler avec son grand-père dans sa langue. Le grandpère avait voulu parler occitan à son petit fils alors qu'il était bébé, mais les parents s'y étaient opposés, arguant que leur enfant risquait de mal apprendre le français. Le reste viendrait après. A raison d'une heure hebdomadaire, il est possible que "le reste" ne vienne que trop tard, à un moment où le grand-père ne sera plus là. Si ce type de situation est courant en Provence, il doit l'être ailleurs, et il aurait été inutile et contre-productif d'essayer de convaincre la mère qu'elle avait eu tort. Mieux vaut, dans ce cas, partir de la situation telle qu'elle se présente.

\subsection{Les élèves : des "néo-locuteurs"?}

Les enfants choisissent souvent eux-mêmes l'option "langue régionale" à l'école, ce qui est d'autant plus méritoire que, les cours étant souvent placés sur l'horaire du déjeuner, ils doivent régulièrement sacrifier une partie de leur repas pour y assister. Les raisons qu'ils invoquent généralement tiennent à la curiosité, à 
la volonté de gagner des points au brevet des collèges ou au baccalauréat, à l'obligation de continuer une option prise plus jeune, au fait que le cours ou l'enseignant sont intéressants, ou à l'impression d'apprendre quelque chose, une dimension souvent sous-estimée. Il ne faut pas oublier qu'en France, suivre une option de langue régionale est un acte volontaire, contraignant, imposant une charge de travail supplémentaire par rapport aux autres élèves, souvent peu gratifiant et très peu reconnu socialement (sur le marché du travail par exemple). Parfois, et c'était le cas dans trois des établissements où j'enseignais, il est impossible de continuer à l'issue du collège - le bénéfice des quatre années d'apprentissage risque alors d'être perdu.

Certains utilisent le terme de "néo-locuteurs" pour désigner ceux qui ont appris la langue hors du milieu familial. Ces enfants seraient classés comme tels selon cette définition. Ce terme, qui n'existe qu'en français ${ }^{27}$, peut parfois avoir des connotations péjoratives. Le néo-locuteur serait alors celui qui parle une néolangue, une langue artificielle - c'est particulièrement le cas en Bretagne, mais aussi en pays d'oc (cf. Blanchet, 2002). Dans cette acception, le terme est peu satisfaisant. La rupture de transmission de la langue ne peut en aucun cas être imputée aux enfants, et une telle conception des néo-locuteurs implique une vision fixiste d'une langue figée dans un passé idéalisé.

Si la majorité des élèves n'avaient pas de volonté de reparler une langue qu'ils n'entendaient alors plus autour d'eux, j'ai pourtant rencontré un nombre relativement élevé de cas que l'on pourrait voir comme atypiques pendant ces quelques années, des élèves parfois très jeunes avec un intérêt inattendu pour la langue et la culture de leur région.

$\mathrm{Ce}$ sont surtout les élèves les plus jeunes qui manifestent le plus d'enthousiasme. Le fait qu'une langue soit associée au passé n'est pas forcément négatif pour eux : cela peut signifier une proximité accrue avec les grands-parents, ou la famille de manière générale. C'est le cas des enfants dont je voudrais maintenant dresser un rapide portrait.

${ }_{27}$ On n'en trouve en effet aucun équivalent ni en anglais, ni en espagnol. 
Ainsi, j'ai eu en cours une élève de $6^{\mathrm{e}}$, que nous appellerons Juliette, qui voulait, disait-elle, retrouver sa langue : elle avait été élevée par ses grands-parents jusqu'à l'âge de six ans, et ils lui avaient parlé en occitan. Si elle l'avait par la suite complètement oublié, elle considérait qu'ayant un jour parlé cette langue, celle-ci lui appartenait de droit.

L'année d'après, Sébastien, un élève de $5^{\mathrm{e}}$ d'un autre établissement, m'a un jour rapporté un enregistrement qu'il avait fait de son grand-père, ce qui a donné l'occasion à l'ensemble de la classe de s'interroger sur la question de la transmission de la langue. Cet enregistrement avait été l'occasion d'échanges sur la vie de son grand-père, mais aussi sur les activités scolaires de Sébastien.

Le cas de Clément, également élève de $5^{\mathrm{e}}$, est lui d'autant plus atypique qu'il souhaitait améliorer un occitan qu'il avait appris avec son grand-père, qui s'occupait souvent de lui, et avec qui il passait la plupart de ses étés. Encouragé par sa famille, il souhaitait pouvoir parler couramment.

C'est le fait d'aller à la chasse avec son père et d'autres hommes du village qui motivait Chloé, d'origine ardéchoise, à apprendre une langue qui s'utilisait encore largement au cours de cette pratique. Je pourrais également citer l'exemple de Carla, dont l'histoire est un peu différente, du fait de sa scolarité en école Calandreta, école associative où l'occitan est la principale langue de scolarisation. D'origine italienne, mais n'ayant pas reçu l'italien en famille, l'occitan faisait figure d'italien de substitution. De fait, c'est une relation passionnelle qu'elle entretenait avec cette langue.

Dans ces cas de figure, l'utilité ou la non-utilité de la langue importe peu. Ces enfants-là ont déjà trouvé leur utilité à la langue, ils savent pourquoi ils viennent en cours, et comprennent d'eux-mêmes que l'enjeu n'est pas scolaire. Ces observations peuvent être rapprochées de celles que fait Boyer (2005), qui note, au sujet des anciens élèves des écoles associatives occitanes Calandretas que, bien qu'ayant oublié la langue, celle-ci en vient à faire partie de leur histoire personnelle, et reste associée à de bons souvenirs. Pour la plupart d'entre eux cependant, au moins en France, les contacts avec la langue régionale sont généralement minimes au sortir de l'école. 
Ainsi, j'ai enseigné l'occitan dans l'enseignement secondaire en Provence pendant trois ans, de 2003 à 2006. D'abord à Marseille, un an, dans un lieu que je considérais alors comme totalement désoccitanisé, mais où il restait un fort substrat linguistique, puis deux ans dans la Drôme, dans un contexte rural où la langue pouvait encore être entendue relativement facilement. Plusieurs questions sont nées de cet enseignement: pourquoi ? pour qui ? quelle(s) variété(s) de langue enseigner? Quelle(s) approches adopter ? S'agissait-il d'adopter une conception patrimoniale de cet enseignement, alors même que je souhaitais participer à la réintroduction d'un réel bilinguisme sociétal ? Et puis, d'autres questions s'y sont ajoutées : pourquoi ces enfants, nombreux au demeurant (près de trente par classe) choisissaient-ils cet enseignement, quitte éventuellement à sacrifier leur pause déjeuner, alors que l'on dit volontiers que les "jeunes" s'investissent peu ? Que cherchaient-ils ? Mais pourquoi n'utilisaient-ils jamais le provençal entre eux? Que signifiaient pour eux cet apprentissage, et cette langue? Autant de questions encore largement sans réponse.

\section{CONCLUSION(S ) : REVITALISATION LINGUISTIQUE ET TRAVAIL DE TERRAIN}

C'est par ses questionnements que je suis venu à la recherche en sociolinguistique sur les pratiques et les discours qui entourent les questions de revitalisation linguistique. J'ai voulu présenter, de manière rétrospective, un travail de terrain atypique, puisqu'il s'agit en fait d'une expérience de pré-terrain, en termes de recherche scientifique en tout cas.

A travers ces quelques portraits d'élèves apprenant l'occitan, qui font pendant aux aspects théoriques de la revitalisation linguistique vu surtout, en Europe, à travers le prisme de la politique linguistique, et qui répondent aux portraits de locuteurs présentés ailleurs dans ce volume, j'ai cherché à montrer deux facettes de ce que peut être un travail de terrain dans de tels contextes : une nécessaire prise en compte des réalités politiques, institutionnelles, et une indispensable attention portée aux voix des acteurs sur le terrain.

La prise en compte des réalités historiques et politiques permet de comprendre le cadre large dans lequel ces pratiques sont mises en œuvre, celui d'une tension 
entre la diversité, nouveau paradigme promu par les instances européennes, et la tentation de l'unique, héritage de l'idéologie qui a vu naître les État-nations au $18^{\mathrm{e}}$ siècle. L'observation micro-sociolinguistique permet quant à elle, de comprendre comment le contexte global est reproduit, négocié ou contesté localement.

La revitalisation linguistique se pose comme une œuvre collective : celle-ci s'appuie sur un récit collectif, qui est négocié entre divers acteurs (militants, linguistes, locuteurs etc.) mais elle implique une multitude de récits personnels. La difficulté consiste à pouvoir faire correspondre les deux types de récits, que le mythe d'une langue, son prestige lié à une littérature ancienne par exemple, puisse faire sens pour les acteurs sociaux hic et nunc, y compris pour les élèves dans les écoles. Le récit des enfants sur la langue, sur leur propre expérience, me semble particulièrement intéressant, en ce sens qu'il montre quels discours circulent dans une communauté imaginée comme l'école, qu'ils sont une étape dans la formation des discours des futurs locuteurs potentiels de cette langue, et enfin qu'ils reflètent les discours des adultes.

Un travail de terrain sur la revitalisation, centré sur les pratiques scolaires, pose de fait également une multitude de problèmes au chercheur. Celui-ci ne devient-il pas automatiquement, quand bien même à son corps défendant, un acteur de la revitalisation? Comment peut-on réfléchir à son articulation avec les acteurs présents sur le terrain, qui attendent de lui une certaine implication, et un certain retour qui puisse les soutenir dans leur action? Comment penser cette implication, inévitable en un sens, la présence du chercheur contribuant à construire l'objet comme valable et digne d'intérêt ? Comment imaginer ce rôle lorsque l'on vient soi-même de la communauté linguistique qui cherche à revitaliser sa langue, sans pour autant apparaître comme un traitre à une cause à laquelle on est associé par ailleurs?

La revitalisation linguistique est, nous l'avons dit, un phénomène complexe, qui met en œuvre diverses idéologies, diverses manières de se représenter au monde, diverses grilles de lecture, dans lesquelles la langue joue un rôle central. Pour ces diverses raisons, un travail de terrain doit être pensé comme un dialogue interdisciplinaire : si le champ des représentations est assez classiquement celui 
des sociolinguistes, celui des idéologies est celui du sociologue, ou de l'anthropologue dans la tradition américaine. Ensemble, ils doivent comprendre quels acteurs sont impliqués, comment s'effectue entre eux la circulation du pouvoir, comment est sélectionnée une variété langagière particulière et pourquoi. Un travail d'analyse linguistique est également nécessaire pour comprendre comment s'opère le changement linguistique, comment les variétés évoluent, et comment une nouvelle variété, que Lafont (1984) nomme "reconstituée", se forme, au sein de divers contextes, et sous l'influence d'acteurs comme les militants, les enseignants, et même, les chercheurs.

Ça n'est que par un travail minutieux d'étude et de comparaison des diverses actions de revitalisation de par le monde, mais également des discours qui accompagnent et fondent ces pratiques, ce que l'on pourrait appeler des récits de revitalisation que nous aboutirons à un tableau plus complet, et à une meilleure compréhension, de ce que revitaliser veut dire dans un monde postmoderne et globalisé.

\section{REFERENCES}

Balibar, R. 1985. L'institution du français : Essai sur le colinguisme des Carolingiens à la République, Paris, Presses Universitaires de France.

Barnes, M. P. 1998. The Norn Language of Orkney and Shetland, Lerwick, Shetland Times.

Blanchet, P. 2002. Langues, cultures et identités régionales en Provence : la métaphore de l'aïoli, Paris, L'Harmattan.

Boyer, H. (dir.) 2005. De l'école occitane à l'enseignement public: vécu et représentations sociolinguistiques. Une enquête auprès d'une groupe d'excalandrons, Paris, L'Harmattan.

Broudic, F. 2000. Qui parle breton aujourd'hui?, Brest, Brud Nevez.

Burban, C. \& Lagarde, C. (dirs.) 2007. L'école, instrument de sauvegarde des langues menacées?, Perpignan, Presses Universitaires de Perpignan. 
Calamel, S. \& Javel, D. 2002. La langue d'oc pour étendard - Les Félibres (18542002), Toulouse, Privat.

Cerquiglini, B. (1999). Les langues de France: Rapport au Ministre de l'Education Nationale, de la Recherche et de la Technologie, et à la Ministre de la Culture et de la Communication. Paris: Institut National de la Langue Française (CNRS).

Cerquiglini, B. (dir.) 2003. Les langues de France, Paris, Presses Universitaires de France.

Costa, J. 2010, à paraître. "'Aviáu enveja de transmetre tres causas" : transmission familiale de l'occitan et idéologies de militants en Provence ', In: Travaux Neuchâtelois de Linguistique, 50.

Costa, J. \& Lambert, P. 2009. 'France and Language(s): Old Policies and New Challenges in Education. Towards a Renewed Framework?' In CIDREE Yearbook: Language policy and practice in Europe - emerging challenges and innovative responses, Brussels, CIDREE/DVO, 15-26.

Council of Europe (1992). European Charter for Regional or Minority Languages. Strasbourg: Council of Europe.

Crystal, D. (2005). Revitalizing the Celtic Languages. XI Annual Conference of the North American Association for Celtic Language Teachers University of Wales, Bangor.

Dorian, N. C. 1981. Language Death: the Life Cycle of a Scottish Gaelic Dialect, Philadelphia, University of Pennsylvania Press.

Eloy, J.-M. 2004a. 'Des langues collatérales : problèmes et propositions' In Des langues collatérales : Problèmes linguistiques, sociolinguistiques et glottopolitiques de la proximité linguistique, dirigé par Eloy, J.-M. (dir.) Paris, L'Harmattan, 5-25.

Eloy, J.-M. (dir.) 2004b. Des langues collatérales : Problèmes linguistiques, sociolinguistiques et glottopolitiques de la proximité linguistique, Paris, L'Harmattan.

Eloy, J.-M. \& Ó hIfearnáin, T. (dirs.) 2007. Langues proches - langes collatérales / Near Languages - Collateral Languages, Paris, L'Harmattan. 
Encrevé, P. 2002. 'La langue de la République', In: Pouvoirs, 100, 123-136.

Gardy, P. 1990. 'Aux origines du discours francophoniste : le meurtre des patois et leur rachat par le français ', In: Langue française, 85, 22-34.

Grin, F. 2003. 'Diversity as Paradigm, Analytical Device, and Policy Goal' In Language Rights and Political Theory, dirigé par Kymlicka, W. (ed.) Oxford \& New York, Oxford University Press, 169-188.

Hale, K., Krauss, M., Watahomigie, L. J., Yamamoto, A. Y., Craig, C., Jeanne, L. M. and England, N. C. 1992. 'Endangered Languages', In: Language, 68, $1,1-42$.

Héran, F., Filhon, A. \& Deprez, C. 2002. 'La dynamique des langues en France au fil du XXe siècle', In: Population \& Sociétés, 376.

Hinton, L. 1996. Flutes of fire : essays on California Indian languages, Berkeley, Heyday Books.

Jaffe, A. 2008. 'Parlers et idéologies langagières', In: Ethnologie française, 38, 3, 517-526.

Kroskrity, P. V. and Field, M. C. (dirs.) 2009. Native American Language Ideologies: Beliefs, Practices and Struggles, Tucson, University of Arizona Press.

Lafont, R. 1984. 'Pour retrousser la diglossie', In: Lengas, 15, 5-36.

Lewis, M. P. (dir.) 2009. Ethnologue: Languages of the World, Dallas, SIL International.

Lodge, R. A. 1993. French: From Dialect to Standard, London, Routledge.

Manzano, F. 2005. 'Les langues régionales de France sont-elles égales dans le recul?', In: Marges linguistiques, 10, 133-156.

Martel, P. 2005. 'Le "patois à l'école"? Retour sur un débat (XIXème-XXème)', In: Marges linguistiques, 10, 301-317.

Martel, P. 2007a. 'Compter les occitanophones... Histoires d'enquêtes' In El discurs sociolingüístic actual català $i$ occità / Lo discors sociolingüistic actual catalan e occitan, dirigé par Czernilofsky, B., Roviró, B., Cichon, P., Hoinkes, U. and Tanzmeister, R. (dirs.), Wien, Praesens Verlag, 201218. 
Martel, P. 2007b. L'école française et l'occitan - Le sourd et le bègue, Montpellier, Presses Universitaires de la Méditerranée.

Mounin, G. 2006 [1992]. 'Discussion - sur la mort des langues' In Pour une linguistique des langues, dirigé par Walter, H. and Feuillard, C. (dirs.), Paris, Presses Universitaires de France, 181-196.

Payton, P. 2000. 'Cornish' In Languages in Britain and Ireland, dirigé par Price, G. (dir.), Oxford, Blackwell, 109-119.

Price, G. (dir.) 2000. Languages in Britain and Ireland, Oxfod, Wiley-Blackwell.

Rulhes, C. 2000. Les Occitans imaginés - ethnicité et prophétisme occitans, Castres, Institut d'Estudis Occitans.

Salminen, T. 2007. 'Europe and North Asia' In Encyclopedia of the World's Endangered Languages, edited by Moseley, C. (dir.) London \& New York, Routledge, 211-280.

Sasse, H.-J. 1992. 'Theory of language death' In Language Death: Factual and Theoretical Explorations with Special Reference to East Africa, dirigé par Brenzinger, M. (dir.) Berlin, New York, Mouton de Gruyter, 59-80.

Sibille, J. 2002. Les Langues Régionales, Paris, Flammarion.

Spolsky, B. 2008. 'Riding the Tiger' In Can Schools Save Indigenous Languages? Policy and Practice on Four Continents, dirigé par Hornberger, N. H. (dir.) Basingstoke \& New York, Palgrave MacMillan, 152-160.

Thomson, R. L. 2000. 'Manx' In Languages in Britain and Ireland, dirigé par Price, G. (dir.) Oxford, Blackwell, 58-69.

Tuaillon, G. 1972. 'Le franco-provençal: progrès d'une définition', In: Travaux de linguistique et et de littérature, 10, 1, 293-339. 